Journal of Communications and Information Networks, Vol.2, No.4, Dec. 2017

DOI: $10.1007 / \mathrm{s} 41650-017-0045-3$

(C) Posts \& Telecom Press and Springer Singapore 2017

Special Focus on Optical Wireless Communication

\title{
Free-space optical channel characterization in a coastal environment
}

\author{
Wael G. Alheadary*, Ki-Hong Park, Boon S. Ooi, Mohamed-Slim Alouini \\ Computer, Electrical, and Mathematical Sciences and Engineering (CEMSE) Division, \\ King Abdullah University of Science and Technology (KAUST), Thuwal, 23955-6900, Saudi Arabia \\ * Corresponding author, Email: wael.headary@kaust.edu.sa
}

\begin{abstract}
Recently, FSO (Free-Space Optical Communication) has received a lot of attention thanks to its high data-rate transmission via unbounded unlicensed bandwidth. However, some weather conditions lead to significant degradation of the FSO link performance. Based on this context and in order to have a better understanding of the capabilities of FSO communication in a coastal environment, the effects of temperature and humidity on an FSO system are investigated in this study. An experiment is conducted using an open source FSO system that achieves a transmission rate of $1 \mathrm{Gbit} / \mathrm{s}$ at a distance of $70 \mathrm{~m}$. Two new mathematical models are proposed to represent the effects of temperature and humidity on our developed FSO system operating at a wavelength of $1550 \mathrm{~nm}$. The first model links the FSO attenuation coefficient to the air temperature in coastal regions, while the second model links the FSO attenuation coefficient to the humidity and the dew-point temperature. The key finding of this study is that FSO links can achieve maximum availability in a coastal city with normal variations in temperature and humidity.
\end{abstract}

Keywords: FSO, optical systems, relative humidity, temperature, channel characterization, attenuation coefficient

\section{Introduction}

Research interest in FSO and UWOC (Underwater Wireless Optical Communication) is increasing rapidly ${ }^{[1]}$. By using a light beam, FSO can transmit and receive data, voice, and multimedia information through the free space in an LOS (Line-of-Sight) position. FSO provides data transmission rates ranging from $100 \mathrm{Mbit} / \mathrm{s}$ to $1.2 \mathrm{Tbit} / \mathrm{s}^{[2]}$. In addition, it is a license-free bandwidth-effective and cost-effective technology compared to RF (Radio Frequency) and optical fiber technologies ${ }^{[3]}$. In cases of bottleneck problems or congestion in a backhaul network, pointto-point or point-to-multipoint FSO has been proposed for use in different topologies as an attractive solution to satisfy the requirements and QoS (Quality of Service) needs of new traffic ${ }^{[4,5]}$.

However, FSO systems suffer from degradation in the link quality owing to several weather conditions $^{[6]}$. Owing to atmospheric variation across the FSO links, many researchers have focused on the visibility between FSO transceivers. For example, the effects of fog and sandstorms were investigated in Ref. [7] based on measurements recorded

Manuscript received Jul. 07, 2017; accepted Aug. 18, 2017

The research reported in this publication was supported by funding from King Abdullah University of Science and Technology (KAUST). 
outdoors in different countries. The impact of fog was investigated through a simulation in a prepared area using a theater smoke machine in Ref. [8]. The work in Ref. [9] focused on the effect of sandstorms. Similarly, Ref. [10] investigated the availability and link budget of FSO channels based on the visibility between the FSO transceivers under different weather conditions. In addition, studies have shown that the optical attenuation coefficient over an FSO link can reach $120 \mathrm{~dB} / \mathrm{km}$ in moderate fog and, $480 \mathrm{~dB} / \mathrm{km}$ in dense fog in winter ${ }^{[11,12]}$. So, as an efficient solution to increase the FSOs links availability and reliability when critical weather conditions arise, hybrid systems have been proposed with a backup link to complement the prime FSO link. For example, in Ref. [13] the researchers measured the availability of a hybrid system comprised of an FSO main link and $40 \mathrm{GHz}$ backup link. The availability of the proposed hybrid system reached $99.92 \%$ compared to the $96.8 \%$ availability of the FSO link alone.

There are many factors that degrade FSO channels depending on the local environment. Consequently, there exists an essential need to investigate the performance of FSO channels under different atmospheric conditions. In this work, we characterize the effect of a coastal environment on FSO channels. In particular, our analysis clarifies whether the temperature and humidity conditions are suitable for FSO systems, and is beneficial to future performance analyses in coastal locations or similar environments. Moreover, this analysis will be beneficial conducting more performance analysis for coastal locations or similar environments. For this purpose, we derived two mathematical models based on our measurements that show the relation between temperature, humidity and link attenuation.

The structure of this paper is as follows: a theoretical analysis of meteorological definitions is derived in section 2, the experimental setup is discussed in section 3 , the experimental results are analyzed and compared with the theoretical analysis in section 4, and the conclusion and future work are discussed in section 5 .

\section{Theoretical analysis of the attenua- tion model}

\subsection{Meteorological analysis}

Humidity is the amount of moisture or water vapor present in the air. Water vapor is the gaseous state of water and is invisible to the human eye as shown in Fig. 1. At the dew point temperature $\left(T_{D}\right)$, the air must be cooled to become saturated with water vapor. In this situation, some of the water vapor must condense to form liquid dew. One way to measure humidity is by using the relative humidity $\left(R_{H}\right) . R_{H}$ is the ratio of actual vapor density to the maximum saturated density, depending on the current air temperature $(T)$. When $R_{H}$ approaches $100 \%$, the air is completely mixed and saturated with water vapor. Consequently, rain will occur. $R_{H}$ can be defined as

$$
R_{H}=\frac{\text { Current absolute humidity }}{\text { Highest absolute humidity }} \times 100 .
$$

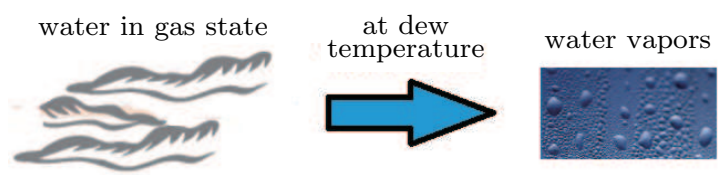

Figure 1 Vapor at dew point when $T_{D}$ is close or equal to normal temperature $T$

At $T_{D}$, the saturated air cools, causing some of the water vapor to condense and become liquid (dew). Many researchers have studied the relations between $R_{H}, T_{D}$, and $T$. There is a very simple approximation that allows for a conversion between the dewpoint temperature, current temperature, and relative humidity:

$$
R_{H}=100-5\left(T-T_{D}\right) .
$$

This approach is accurate to within about $\pm 1^{\circ} \mathrm{C}$ as long as the relative humidity is above $50 \%^{[13]}$. A more accurate equation from Ref. [14] represents the relative humidity in terms of $T_{D}$ and $T$ :

$$
R_{H}=\frac{\exp \left(17.625 \times T_{D} /\left(243.04+T_{D}\right)\right)}{\exp (17.625 \times T /(243.04+T))} \times 100 .
$$

The following model is proposed in Ref. [15]:

$$
R_{H}=K \frac{T_{D}}{T} \times 100,
$$


where $K$ is constant. Based on our measurements and Ref. [15], $K=0.97$.

All of proposed models show an inverse relation between $R_{H}$ and $T$, which will be explained in the experimental results and analysis section.

\subsection{FSO attenuation in terms of coastal temperature}

To the best of our knowledge, no previous work has characterized the effect of temperature in a coastal environment. Based on Ref. [10], if $\gamma$ is the link attenuation coefficient in $\mathrm{dB} / \mathrm{km}$ and $T$ is the air temperature, the attenuation coefficient is always inversely proportional to the temperature at that time. Therefore, the relation between $T$ and $\gamma$ can be modeled as

$$
\frac{\mathrm{d} \gamma}{\mathrm{d} T} \propto(-\gamma) .
$$

With some manipulation, we represent Eq. (5) as

$$
\frac{\mathrm{d} \gamma}{\gamma}=-b \mathrm{~d} T
$$

where the negative sign represents the inverse relationship between $\gamma$ and $T$, and $b$ is constant. Integrating both sides of Eq. (6) within the temperature range ( 0 to $T$ ) leads to

$$
\int_{\gamma_{0}}^{\gamma} \frac{\mathrm{d} \gamma}{\gamma}=-\int_{0}^{T} b \mathrm{~d} T
$$

Thus, the general model linking the temperature to the attenuation coefficient can be written as

$$
\gamma=c \mathrm{e}^{-b T},
$$

where $c=1 / \gamma_{0}$ is a constant. The constants $c$ and $b$ can be obtained for any temperature from the experimental measurements. In addition, $c$ and $b$ are wavelength-dependent.

\subsection{FSO attenuation in terms of coastal relative humidity}

Moisture in the atmosphere will absorb some of the transmitted optical power, depending on the volume and quantity of water vapor present in the air. To derive a model that shows the relation between the FSO link attenuation coefficient and the relative humidity, we insert Eqs. (2)-(4) into Eq. (8), leading to

Model 1: $\gamma=c \mathrm{e}^{-b\left(T_{D}+\left(100-R_{H}\right) / 5\right)}$,

Model 2:

$$
\begin{aligned}
\gamma=c \exp [-b 243.04 & {\left[\begin{array}{l}
\frac{17.625 \times T_{D}}{243.04+T_{D}}-\log \left(\frac{R_{H}}{100}\right) \\
\times \\
17.625+\log \left(\frac{R_{H}}{100}\right)-\frac{17.625 \times T_{D}}{243.04+T_{D}}
\end{array}\right], }
\end{aligned}
$$

Model 3: $\gamma=c \mathrm{e}^{-b K T_{D} / R_{H}}$.

In the experimental results and analysis section, we will investigate the best model for a coastal environment in terms of the RMSE (Root Mean Square Error) and the goodness of fit $R^{2}$.

\section{$3 \quad$ Experimental setup}

We designed and integrated a complete mobile FSO system. This system was deployed in less than an hour. The system consists of two carts, and each cart consists of a breaking system, two Koruza units, wireless modems with data SIM (Subscriber Identification Module) cards, a weather station, a deep cycle battery or solar panel system, and mounts for the optical beam alignment. The Koruza system is an open-source, low-cost FSO system that is designed to be upgradable ${ }^{[16]}$. A detailed description of the system and its integration is presented in Fig. 2(a).

The mobile FSO system was tested in an outdoor environment. The weather is typically warm and wet with minimal variations in temperature at KAUST (King Abdullah University of Science and Technology), because it is located near the Red Sea, in Thuwal, Saudi Arabia as shown in Fig. 2(b). Minimal variations in temperature make coastal cities suitable for utilizing FSO systems. We recorded the link measurements and weather data during August 2016, since the humidity and temperature were the highest during this year. 


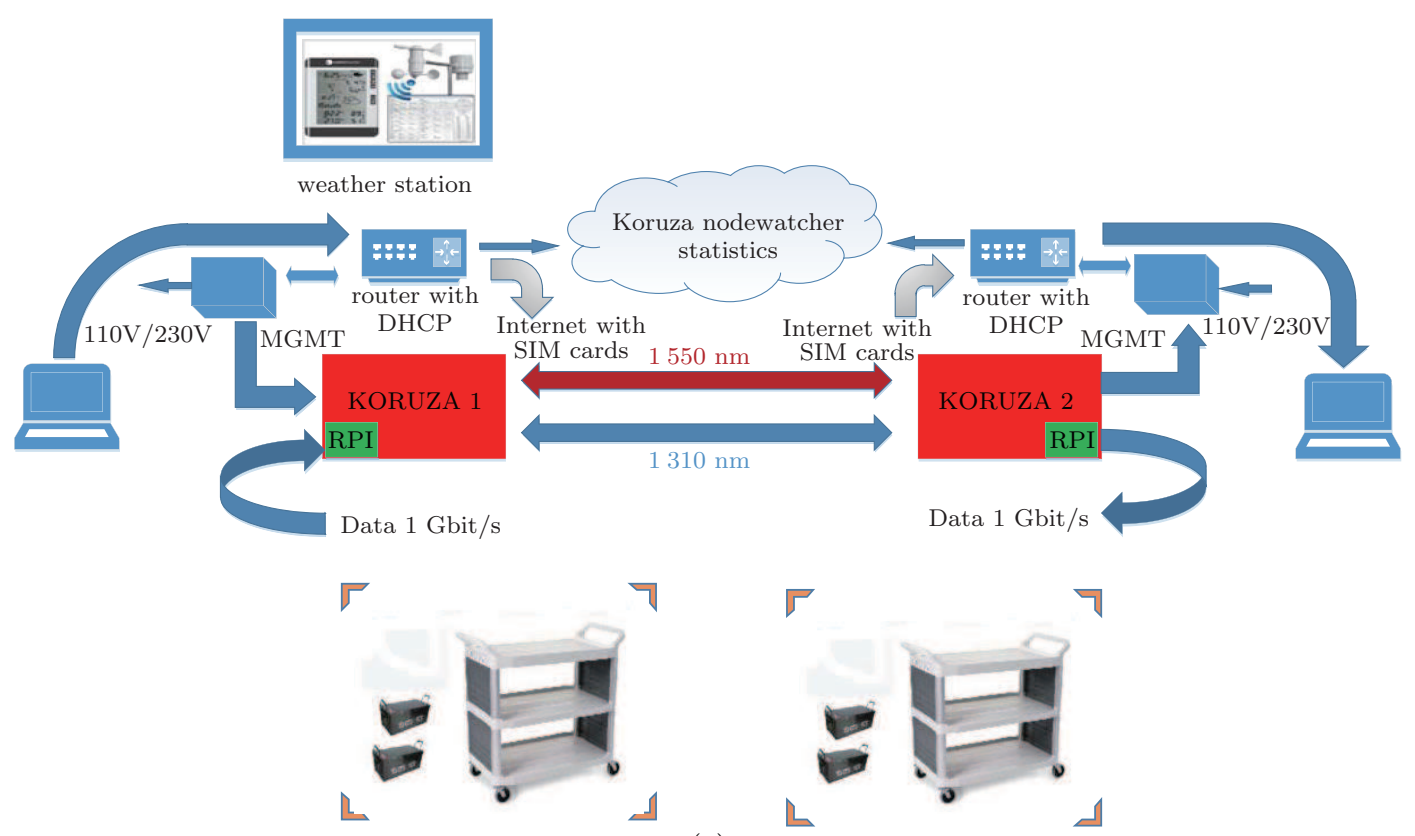

(a)

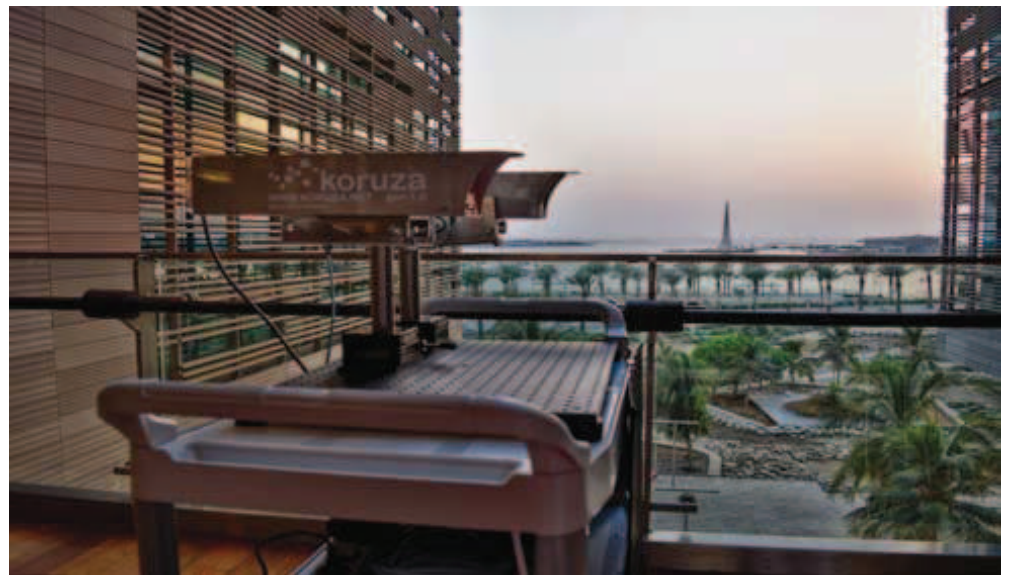

(b)

Figure 2 Proposed mobile FSO system:

(a) system design; (b) coastal environment

The fluctuation of link quality was monitored in the outdoor case using the Koruza system. The weather parameters were measured using a Ws-2095 weather station, which was installed next to the FSO transceivers. We measured the temperature, relative humidity, and dew-point temperature. As shown in Fig. 2(a), the received power was uploaded to a predefined server via the wireless modem, and the temperature, dew-point temperature, and relative humidity data were recorded by the weather station data logger. The FSO system and weather station data were collected every $10 \mathrm{~min}$.

\section{Experimental results and analysis}

To validate our work, we show the relation between the atmospheric attenuation coefficient $(1 / \mathrm{km})$ and the weather station data. The attenuation coefficient is computed by measuring the variation between the maximum received power and the instantaneous received power in decibels $(\mathrm{dB})$ over the transmission distance in kilometers $(\mathrm{km})$.

Fig. 3 demonstrates an inverse relationship between the relative humidity and the temperature. We filtered out unreliable link measurements due to 
thermal expansion from the summer heat and alignment issues.

The relationship between the measured temperature and the link attenuation coefficient is represented in Fig. 4; in this case, the attenuation coefficient changes inversely with the temperature. Based on the measurements of link attenuation and temperature, we applied a curve-fitting method and found the exponential attenuation model, $\gamma=$ $20.82 \mathrm{e}^{-0.03115 T}$. This model shows an accurate fit to the experimental measurements with $R M S E=$ 0.0011 and $R^{2}=0.9997$.

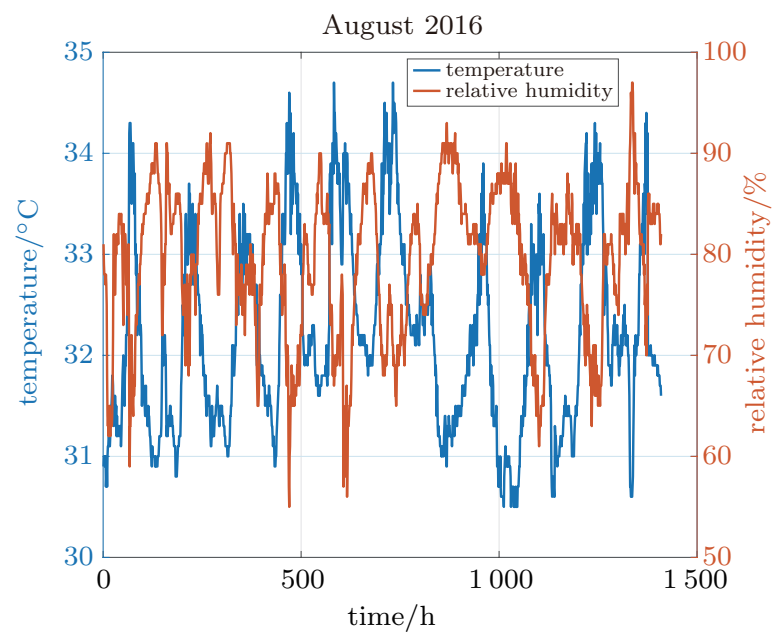

Figure 3 Temperature and relative humidity

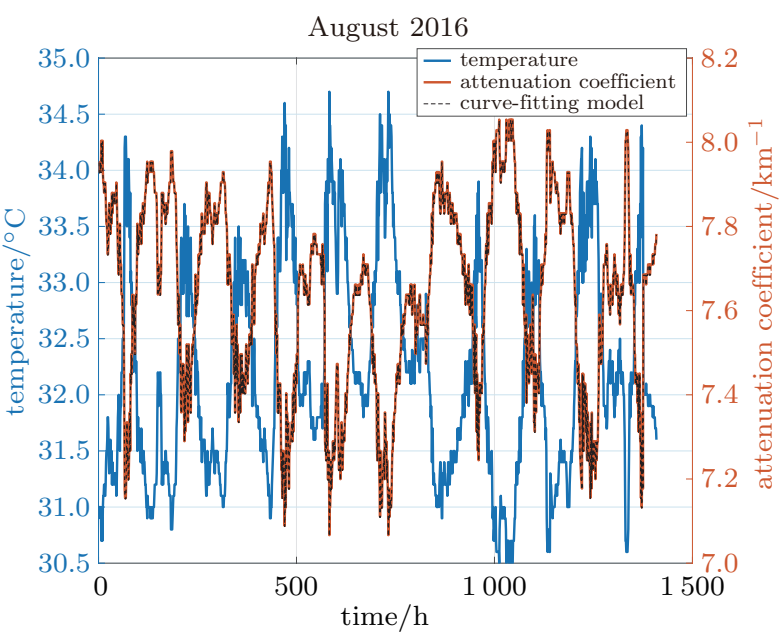

Figure 4 Relation between temperature and attenuation coefficient

Fig. 5 verifies the attenuation coefficient values obtained from the curve-fitting model with the mea- sured values of attenuation.

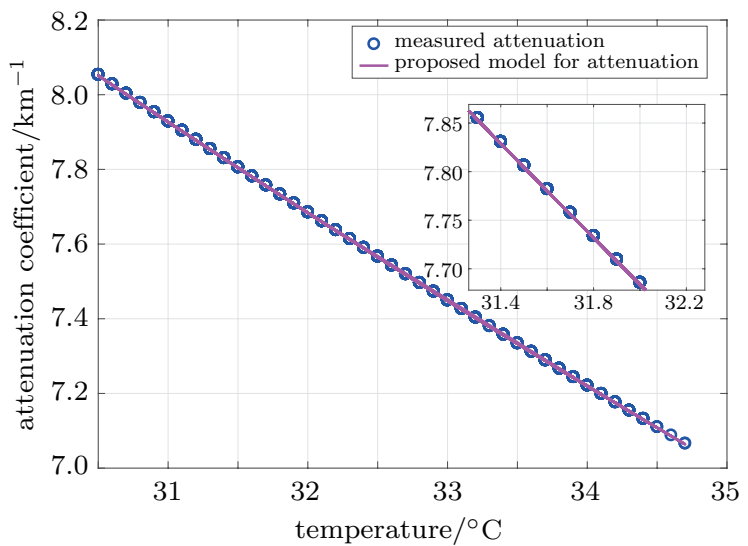

Figure 5 Verification of our attenuation model

On the other hand, Fig. 6 shows that the measured attenuation coefficient has a direct relationship with changes in humidity. Hence, the attenuation coefficient increases with high humidity measurements.

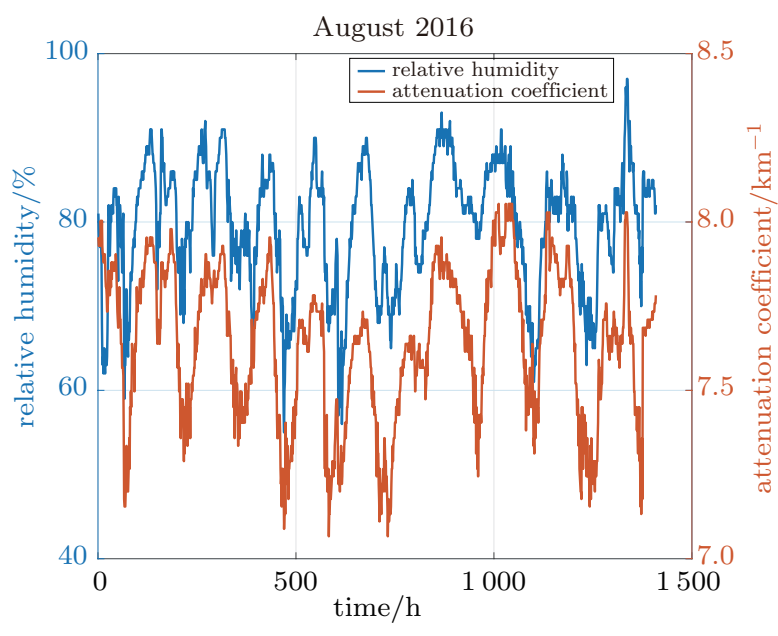

Figure 6 Relative humidity and attenuation coefficient

In order to derive a model of the relation between the relative humidity and the attenuation coefficient, we will compare Eqs. (9)-(11) with the measured attenuation coefficient. As seen in Tab. 1, Model 2 is more accurate than the other models.

In addition, the relation between the measured attenuation and the calculated attenuation models is shown graphically in Fig. 7, to show the accuracy of the attenuation model. We conclude the small blue circles have a good linear relation with the measured 
data. It was difficult to determine the direct relationship between the relative humidity and the attenuation coefficient, because there were many factors including pressure, wind, etc. that could affect the humidity measurements. In our work, we measured both the dew-point temperature and the relative humidity to obtain an accurate attenuation and humidity model.

Table 1 Values of fitting parameters with measured attenuation models from Eqs. (9)-(11)

\begin{tabular}{ccc}
\hline model & RMSE & $R^{2}$ \\
\hline 1 & 0.1888 & 0.9625 \\
2 & 0.0483 & 0.9975 \\
3 & 0.3456 & 0.8744 \\
\hline
\end{tabular}

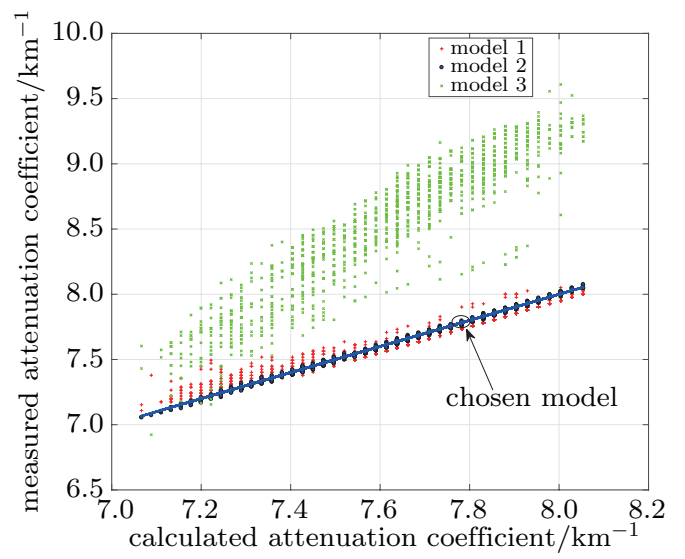

Figure 7 Relation between measured and calculated attenuation coefficients based on $R_{H}$ and $T_{D}$

Therefore, we conclude that variations in weather conditions impact the FSO link fluctuation significantly.

\section{Conclusion and future work}

In this work, we proposed two mathematical models that focus on the relations between temperature, relative humidity, and the atmospheric attenuation coefficient over FSO links in coastal regions. In addition, we designed and integrated an open-source and mobile FSO system that can be easily deployed and applied in a variety of environments. The time for deployment was less than one hour, depending on the distance. This system showed reliable mobile FSO links at a transmission distance of $70 \mathrm{~m}$. Furthermore, this study shed more light on how humidity and temperature significantly affect FSO links. The proposed models showed a good fit with the measured attenuation. The introduced system is being continuously improved to make it sustainable and reliable in an extreme environment. In addition, we are working on models to clarify the effects of weather conditions e.g. humidity, pressure, rain, and wind over optical links with different wavelengths.

\section{References}

[1] C. Shen, Y. Guo, H. Oubei, et al. 20-meter underwater wireless optical communication link with $1.5 \mathrm{Gbps}$ data rate [J]. Optics express, 2016, 22(24): 25502-25509.

[2] H. Hogan. Data demands drive free-space optics [J]. Photon. spectra., 2013, 2: 32-48.

[3] L. Andrews, R. Phillips, C. Hopen. Laser beam scintillation with applications [C]//SPIE Publications, 2001.

[4] S. Al-Gailani, A. Mohammad, R. Shaddad. Enhancement of free space optical link in heavy rain attenuation using multiple beam concept [J]. Optik-international journal for light and electron optics, 2013, 124(21): 4798-4801.

[5] C. C. Davis, I. I. Smolyaninov, S. D. Milner. Flexible optical wireless links and networks [J]. IEEE commun. mag., 2003, 41(3): 51-57.

[6] H. Henniger, O. Wilfert. An introduction to free-space optical communications [J]. Radio engineering, 2010, 19(2): 203-212.

[7] M. Esmail, H. Fathallah, M. S. Alouini. Outdoor FSO communications under fog: attenuation modeling and performance evaluation [J]. IEEE photonics journal, 2016, 8(4): 2894-2903.

[8] M. Esmail, H. Fathallah, M. S. Alouini. An experimental study of FSO link performance in desert environment [J]. IEEE commun. Lett., 2016, 20(9): 1888-1891.

[9] L. Mustafa, E. Cerncic, B. Thomsen. FSO artificial low-cost fog attenuation experiment design [C]//International Symposium on Communication Systems, Networks and Digital Signal Processing (CSNDSP), Prague, 2016.

[10] A. Prokes. Atmospheric effects on availability of free space optics systems [J]. Optical engineering, 2009, 48(6): 066001.

[11] M. S. Khan, S. S. Muhammad, M. S. Awan, et al. Further results on fog modeling for terrestrial free-space optical links [J]. Opt. eng., 2012, 51(3): 1-9.

[12] S. S. Muhammad, B. Flecker, E. Leitgeb, et al. Characterization of fog attenuation in terrestrial free space optical links [J]. Optical engineering, 2007, 46(6): 066001. 
[13] M. G. Lawrence. The relationship between relative humidity and the dewpoint temperature in moist air: a simple conversion and applications $[\mathrm{J}]$. Bulletin of the American Meteorological Society, 2005, 86(2): 225-233.

[14] O. A. Alduchov, R. E. Eskridge. Improved Magnus form approximation of saturation vapor pressure [J]. Appl. meteor., 1996, 35: 601-609.

[15] S. A. Al-Gailani, A. B. Mohammad, M. S. Islam, et al. Tropical temperature and humidity modeling for free space optical link [J]. J. opt., 2015, 45(1): 87-91.

[16] L. Mustafa. Light speed networking [EB/OL]. http: //www.koruza.net/.

\section{About the authors}

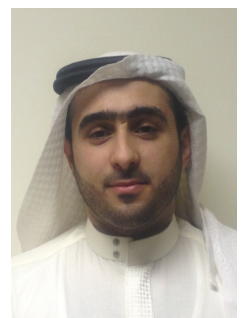

Wael G. Alheadary [corresponding author] received his B.Sc. and M.S. degrees in computer engineering from King Saud University, Riyadh, Saudi Arabia, in 2005 and 2009 respectively. In 2005, he joined King Abdulaziz City for Science and Technology (KACST) as a research engineer. In 2013, he joined Taiba University in Madinah, Saudi Arabia, as a lecturer. He got a scholarship the same year to continue his Ph.D. degree in electrical engineering in the Division of Computer, Electrical, Mathematical Science and Engineering (CEMSE) at King Abdullah University of Science and Technology (KAUST), Thuwal, Saudi Arabia. His current research interests include the modeling, design, and performance analysis of optical wireless communication systems. (Email: wael.headary@kaust.edu.sa)

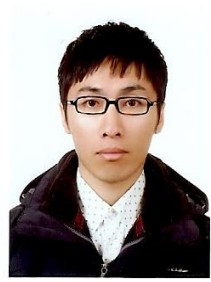

Ki-Hong Park received his B.Sc. degree in electrical, electronic, and radio engineering from Korea University, Seoul, Korea, in 2005 and his M.S. and Ph.D. degrees in the School of Electrical Engineering from Korea University, Seoul, Korea, in 2011. Since 2011, he has been a postdoctoral fellow of electrical engineer- ing in the Division of Computer, Electrical, Mathematical Science and Engineering (CEMSE) at King Abdullah University of Science and Technology (KAUST), Thuwal, Saudi Arabia. He is currently working as a research scientist of CEMSE at KAUST. His research interests are broad in communication theory and its application to the design and performance evaluation of wireless communication systems and networks. His on-going research includes the application to MIMO diversity/beamforming systems, physical layer secrecy, and optical wireless communications. (Email:kihong.park@kaust.edu.sa)

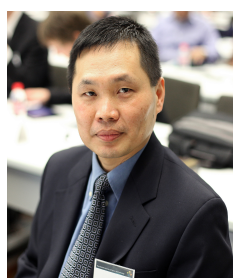

Boon S. Ooi is a professor of electrical engineering at KAUST. He received his Ph.D. degree from the University of Glasgow (UK) in 1994. He joined KAUST from Lehigh University (USA) in 2009. His recent research is concerned with the study of GaN-based materials and devices, and lasers for applications such as solid-state lighting, visible light and underwater wireless optical communications. He currently serves on the editorial board of Journal of nanophotonics and optics express. He is a fellow of SPIE, a fellow of the Institute of Physics (UK), and a senior member of IEEE. (Email:boon.ooi@kaust.edu.sa)

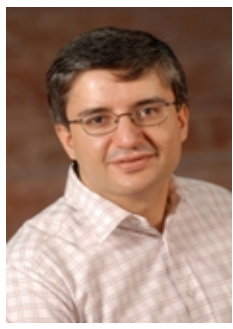

Mohamed-Slim Alouini was born in Tunis, Tunisia. He received his Ph.D. degree in electrical engineering from the California Institute of Technology (Caltech), Pasadena, CA, USA, in 1998. He served as a faculty member in the University of Minnesota, Minneapolis, MN, USA, then in the Texas A\&M University at Qatar, Education City, Doha, Qatar before joining King Abdullah University of Science and Technology (KAUST), Thuwal, Makkah Province, Saudi Arabia, as a professor of electrical engineering in 2009. His current research interests include the modeling, design, and performance analysis of wireless communication systems. (Email:slim.alouini@kaust.edu.sa) 